\title{
Nonlinear acoustic propagation in two-dimensional ducts
}

\author{
Ali Hasan Nayfeh and Ming-Shing Tsai \\ Virginia Polytechnic Institute and State University, Blacksburg, Virginia 24061
}

(Received 7 May 1973; revised 18 December 1973)

\begin{abstract}
The method of multiple scales is used to obtain a second-order uniformly valid expansion for the nonlinear acoustic wave propagation in a two-dimensional duct whose walls are treated with a nonlinear acoustic material. The wave propagation in the duct is characterized by the unsteady nonlinear Euler equations. The results show that nonlinear effects tend to flatten and broaden the absorption versus frequency curve, in qualitative agreement with the experimental observations. Moreover, the effect of the gas nonlinearity increases with increasing sound frequency, whereas the effect of the material nonlinearity decreases with increasing sound frequency.
\end{abstract}

Subject Classification: $25.20 ; 20.45 ; 25.22 ; 20.15$.

\section{INTRODUCTION}

Measured data in typical jet engines indicate that the sound pressure levels involved may be in excess of 160 $\mathrm{dB}$ which corresponds to a pressure fluctuation of the order of $0.01 \mathrm{~atm}$. At these levels, the nonlinear effects play an important role in the attenuation of the sound. These nonlinear effects can be classified into two types: the nonlinearity of the gas itself and the nonlinearity of the acoustic properties of the lining material. Although the gas nonlinearity is significant at sound pressure levels exceeding $160 \mathrm{~dB}$, the material nonlinearity (especially perforated plates) may be significant at sound pressure levels above $130 \mathrm{~dB}$.

Most of the studies of the nonlinear effects of the gas have been based on an irrotational, inviscid, compressible wave propagating in a hard-walled uniform duct with no mean flow. Fay ${ }^{1}$ obtained a Fourier series solution, valid in the farfield, for the one-dimensional wave equation taking into account the effects of gas compressibility and viscosity. Fubini ${ }^{2}$ obtained a solution, valid in the nearfield, for the one-dimensional, isentropic wave equation in terms of Bessel functions. Blackstock ${ }^{3}$ used the weak shock theory to obtain a general solution which is valid in the near- and farfields as well as the transition between them. Coppens ${ }^{4}$ and Pestorius and Blackstock ${ }^{5}$ determined the viscous and thermal dissipative effects on the nonlinear propagation of plane waves in hard-walled ducts.

Maslen and Moor ${ }^{6}$ used the method of strained parameters (e.g., Sec. 3.1 of Ref. 7) to analyze strong transverse waves in a circular cylinder. They determined the effect of the amplitude on the frequency of oscillation. They also determined the dissipative effects of the acoustic boundary layer. Burns ${ }^{8}$ analyzed finiteamplitude waves in a hard-walled duct, taking into account dissipation. However, his expansion is not uniformly valid because it contains secular terms. Keller and Millman ${ }^{9}$ used the method of strained parameters to determine the wavenumber shift for the dispersive modes in a hard-walled duct. Peube and Chasseriaux ${ }^{10}$ treated nonlinear wave propagation in hard-walled ducts with variable cross section. They proposed two expansions, one valid for small Mach numbers and the other valid for long waves. However, no solutions were obtained to assess the nonlinear effects.
Zorumski and Parrott ${ }^{11}$ and Kurze and Allen ${ }^{12}$ found, experimentally, that the nonlinear material effects tend to flatten and broaden the absorption versus frequency curve. At resonance, the high intensity level increases the resistance of the liner resulting in a lower attenuation. To quantify the nonlinear effects on the attenuation, Ingard ${ }^{13,14}$, used the one-dimensional transmission line approximation to determine the nonlinear material effect on the attenuation of the lowest mode at low frequencies. Kurze and Allen ${ }^{12}$ extended the work of Ingard to the region of resonance. They found that their analytical solution is in agreement with their experimental results.

Isakovich ${ }^{15}$ determined a second-order expansion for the nonlinear motion of an irrotational, inviscid, compressible gas in a duct lined with a material having linear acoustic properties. He found that the expansion is free of secular terms as opposed to the case of propagation in an unbounded space.

The purpose of the present paper is to analyze the nonlinear effects of both the gas motion and the lining material on the propagation and attenuation of all modes in a uniform two-dimensional duct by determining a third-order uniform expansion using the method of multiple scales (e.g., Chap. 6 of Ref. 7).

\section{PROBLEM FORMULATION}

We consider nonlinear acoustic wave propagation in a uniform two-dimensional duct whose walls are lined with an acoustic material. We assume the gas to be inviscid, irrotational, and initially quiescent with a uniform pressure $p_{0}$ and a uniform density $\rho_{0}$. The limitations of the inviscid, irrotational assumption will be discussed below. We introduce a Cartesian coordinate system whose $x$ axis coincides with the center of the duct, and its $y$ axis is normal to the walls of the duct (see Fig. 1). We introduce dimensionless quantities using the ambient speed of sound $c_{0}$, the half-width $d$ of the duct, and the ambient density $\rho_{0}$. Thus, we let $\overrightarrow{\mathbf{r}}=\overrightarrow{\mathrm{r}} * / d, \overrightarrow{\mathrm{v}}=\overrightarrow{\mathrm{v}} * / c_{0}, \rho=\rho^{*} / \rho_{0}, p=p^{*} / \rho_{0} c_{0}^{2}$, and $t=t^{*} c_{0} / d$, where starred and unstarred quantities denote dimensional and dimensionless quantities, respectively, $\vec{r}$ is the position vector, $\vec{v}$ is the velocity vector, $\rho$ is the gas density, $p$ is the gas pressure, and $t$ is the time. In terms of these dimensionless quantities, the equations 


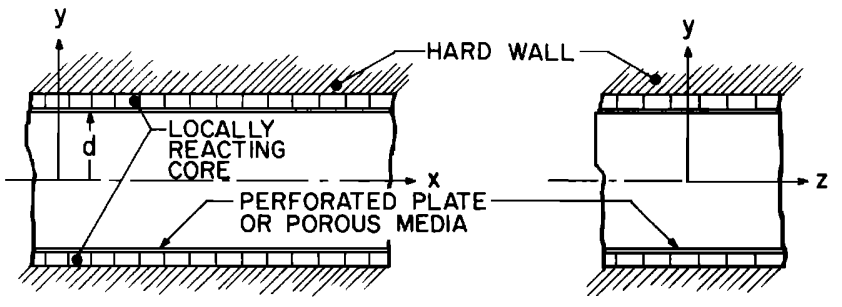

FIG. 1. A schematic of the flow configuration.

describing the conservation of mass and momentum are

$$
\begin{aligned}
& \frac{\partial \rho}{\partial t}+\nabla \cdot(\rho \overrightarrow{\mathrm{v}})=0, \\
& \rho\left(\frac{\partial \overrightarrow{\mathrm{v}}}{\partial t}+\overrightarrow{\mathrm{v}} \cdot \nabla \overrightarrow{\mathrm{v}}\right)=-\nabla p+\frac{1}{\operatorname{Re}} \nabla \cdot \underline{\underline{\tau}},
\end{aligned}
$$

where $\tau$ is the dimensionless stress tensor and Re $=c_{0} d / \nu$ with $\nu$ the kinematic viscosity of the gas. In what follows, we neglect the viscous terms in Eq. 2 and assume that $\overrightarrow{\mathrm{v}}$ is derivable from the dimensionless potential function $\phi(x, y, t)$; that is,

$$
\overrightarrow{\mathrm{v}}=\nabla \phi \text {. }
$$

Moreover, we assume that the pressure and density are related by the isentropic relationship

$$
p * / p_{0}=\left(\rho * / \rho_{0}\right) \gamma
$$

or, in dimensionless quantities, by

$$
\gamma p=\rho^{\gamma},
$$

where $\gamma$ is the specific heat ratio of the gas.

Substituting for $\overrightarrow{\mathrm{v}}$ and $p$ from Eqs. 3 and 4 into Eq. 2 with $\mathrm{Re}=\infty$ and integrating, we obtain

$$
\rho^{\gamma-1}=(1-\gamma)\left[\phi_{t}+\frac{1}{2}(\nabla \phi)^{2}\right]+1
$$

or, in two-dimensional form,

$$
c^{2}=(1-\gamma)\left[\phi_{t}+\frac{1}{2}\left(\phi_{x}^{2}+\phi_{y}^{2}\right)\right]+1,
$$

where $c$ is the speed of sound which is related to $p$ and $\rho$ by

$$
c^{2}=\gamma p / \rho=\rho^{\gamma-1} .
$$

Differentiating Eq. 5 with respect to $t$, eliminating $\rho$ using Eqs. 1, 3, 5, and 7, and arranging, we obtain

$$
\frac{\partial^{2} \phi}{\partial t^{2}}+\frac{\partial}{\partial t}(\nabla \phi)^{2}+\frac{1}{2} \nabla \phi \cdot \nabla(\nabla \phi)^{2}=c^{2} \nabla^{2} \phi
$$

or, in two-dimensional form,

$$
\begin{aligned}
\phi_{x x}+\phi_{y y}-\frac{1}{c^{2}} & {\left[\phi_{t t}+\frac{\partial}{\partial t}\left(\phi_{x}^{2}+\phi_{y}^{2}\right)+\phi_{x x} \phi_{x}^{2}\right.} \\
& \left.+\phi_{y y} \phi_{y}^{2}+2 \phi_{x} \phi_{y} \phi_{x y}\right]=0 .
\end{aligned}
$$

We assume the walls to be acoustically treated by a liner consisting of a thin rigid porous sheet or perforated plate backed by honeycomb cavities. Several forms have been proposed for the nonlinear impedance of such a liner (see, for example, Melling ${ }^{16}$ for a review and new results). In this paper, we use the following semiempirical form proposed by Zorumski and Parrott ${ }^{17}$ :

$$
p^{*}-p_{0}:\left[\mathrm{R}^{*}\left(\mathrm{v}^{*}\right)+\chi^{*}\left(\mathrm{v}^{*}, \omega^{*}\right)\left(\frac{\partial}{\partial t^{*}}\right)\right] \mathrm{v}^{*}
$$

where $R^{*}$ is the nonlinear resistance, $\chi^{*}$ is related to the nonlinear reactance, and $p^{*}$ and $v^{*}$ are, respective$\mathrm{ly}$, the pressure and normal velocity at the inner duct walls. This form is based on what Zorumski and Parrott call the "instantaneous acoustic laws," which imply incompressibility across the sheet or plate and include a semiempirical term in the momentum equation.

For simplicity, we assume that both walls are lined with the same material, so that we consider the upper half of the duct. Moreover, the acoustic disturbances are either symmetric or antisymmetric. We consider the symmetric case in this paper; that is,

$$
\phi_{y}(x, 0, t)=0 \text {. }
$$

In terms of the dimensionless quantities defined above, we rewrite Eq. 10 as

$$
p-\gamma^{-1}=\left[R\left(\phi_{y}\right)+\chi\left(\phi_{y}\right) \frac{\partial}{\partial t}\right] \phi_{y}, \text { at } y=1,
$$

where $R$ is the nonlinear dimensionless resistance and $\chi$ is related to the nonlinear dimensionless reactance. In what follows, we assume that $R$ and $\chi$ are analytic functions of $\phi_{y}$ and hence expand them as even polynomials in $\phi_{y}$. Eliminating $p$ and $\rho$ from Eqs. 6, 7, and 12 and using the polynomial expansions of $R$ and $\chi$, we obtain

$$
\begin{gathered}
-\phi_{t}-\frac{1}{2}\left(\phi_{x}^{2}+\phi_{y}^{2}\right)+\frac{1}{2} \phi_{t}^{2}+\frac{1}{2} \phi_{t}\left(\phi_{x}^{2}+\phi_{y}^{2}\right)-\frac{1}{6}(2-\gamma) \phi_{t}^{3} \\
=\left[R_{0}+R_{2} \phi_{y}^{2}+\left(\chi_{0}+\chi_{2} \phi_{y}^{2}\right) \frac{\partial}{\partial t}\right] \phi_{y}+0\left(\phi^{4}\right) \text {, at } y=1 .
\end{gathered}
$$

To determine an approximate solution for Eqs. 6 and 9 subject to the boundary conditions 11 and 13, we use the method of multiple scales (see, for example, Chap. 6 of Ref. 7) and let

$$
\phi=\sum_{n=1}^{3} \epsilon^{n} \phi_{n}\left(x_{0}, x_{2}, y, t\right)+0\left(\epsilon^{4}\right),
$$

where $\epsilon$ is a small but finite parameter characterizing the amplitude of the wave, and $x_{0}=x$ is a fast scale characterizing the wavelength while $x_{2}=\epsilon^{2} x$ is a slow scale characterizing the amplitude modulation. The axial derivative becomes

$$
\partial / \partial x=\partial / \partial x_{0}+\epsilon^{2} \partial / \partial x_{2} \cdot
$$

Substituting Eqs. 14 and 15 into Eqs. 6, 9, 11, and 13 and equating coefficients of like powers of $\epsilon$, we obtain:

\section{order $\epsilon$ :}

$$
\begin{aligned}
& \frac{\partial^{2} \phi_{1}}{\partial x_{0}^{2}}+\frac{\partial^{2} \phi_{1}}{\partial y^{2}}-\frac{\partial^{2} \phi_{1}}{\partial t^{2}}=0, \\
& \partial \phi_{1} / \partial y=0, \text { at } y=0, \\
& \frac{\partial \phi_{1}}{\partial t}+R_{0} \frac{\partial \phi_{1}}{\partial y}+\chi_{0} \frac{\partial^{2} \phi_{1}}{\partial y \partial t}=0, \text { at } y=1 .
\end{aligned}
$$


order $\epsilon^{2}:$

$\frac{\partial^{2} \phi_{2}}{\partial x_{0}^{2}}+\frac{\partial^{2} \phi_{2}}{\partial y^{2}}-\frac{\partial^{2} \phi_{2}}{\partial t^{2}}=(\gamma-1) \frac{\partial \phi_{1}}{\partial t} \frac{\partial^{2} \phi_{1}}{\partial t^{2}}+\frac{\partial}{\partial t}\left[\left(\frac{\partial \phi_{1}}{\partial x_{0}}\right)^{2}+\left(\frac{\partial \phi_{1}}{\partial y}\right)^{2}\right]$,

$\partial \phi_{2} / \partial y=0$, at $y=0$,

$\frac{\partial \phi_{2}}{\partial t}+R_{0} \frac{\partial \phi_{2}}{\partial y}+\chi_{0} \frac{\partial^{2} \phi_{2}}{\partial y \partial t}=\frac{1}{2}\left(\frac{\partial \phi_{1}}{\partial t}\right)^{2}-\frac{1}{2}\left(\frac{\partial \phi_{1}}{\partial x_{0}}\right)^{2}-\frac{1}{2}\left(\frac{\partial \phi_{1}}{\partial y}\right)^{2}$,

at $y=1$.

order $\epsilon^{3}:$

$$
\begin{gathered}
\frac{\partial^{2} \phi_{3}}{\partial x_{0}^{2}}+\frac{\partial^{2} \phi_{3}}{\partial y^{2}}-\frac{\partial^{2} \phi_{3}}{\partial t^{2}}=-2 \frac{\partial^{2} \phi_{1}}{\partial x_{0} \partial x_{2}}+(\gamma-1)\left\{\frac{\partial \phi_{2}}{\partial t} \frac{\partial^{2} \phi_{1}}{\partial t^{2}}\right. \\
\left.+\frac{\partial \phi_{1}}{\partial t}\left(\frac{\partial^{2} \phi_{2}}{\partial x_{0}^{2}}+\frac{\partial^{2} \phi_{2}}{\partial y^{2}}\right)+\frac{1}{2} \frac{\partial^{2} \phi_{1}}{\partial t^{2}}\left[\left(\frac{\partial \phi_{1}}{\partial x_{0}}\right)^{2}+\left(\frac{\partial \phi_{1}}{\partial y}\right)^{2}\right]\right\} \\
+2 \frac{\partial}{\partial t}\left(\frac{\partial \phi_{1}}{\partial x_{0}} \frac{\partial \phi_{2}}{\partial x_{0}}+\frac{\partial \phi_{1}}{\partial y} \frac{\partial \phi_{2}}{\partial y}\right)+\frac{\partial^{2} \phi_{1}}{\partial x_{0}^{2}}\left(\frac{\partial \phi_{1}}{\partial x_{0}}\right)^{2} \\
+\frac{\partial^{2} \phi_{1}}{\partial y^{2}}\left(\frac{\partial \phi_{1}}{\partial y}\right)^{2}+2 \frac{\partial \phi_{1}}{\partial x_{0}} \frac{\partial \phi_{1}}{\partial y} \frac{\partial^{2} \phi_{1}}{\partial x_{0} \partial y}
\end{gathered}
$$

$\partial \phi_{3} / \partial y=0$, at $y=0$,

$$
\begin{aligned}
\frac{\partial \phi_{3}}{\partial t}+R_{0} & \frac{\partial \phi_{3}}{\partial y}+\chi_{0} \frac{\partial^{2} \phi_{3}}{\partial y \partial t}=-\frac{\partial \phi_{1}}{\partial x_{0}} \frac{\partial \phi_{2}}{\partial x_{0}}-\frac{\partial \phi_{1}}{\partial y} \frac{\partial \phi_{2}}{\partial y} \\
+ & \frac{1}{2} \frac{\partial \phi_{1}}{\partial t}\left[2 \frac{\partial \phi_{2}}{\partial t}+\left(\frac{\partial \phi_{1}}{\partial x_{0}}\right)^{2}+\left(\frac{\partial \phi_{1}}{\partial y}\right)^{2}-\frac{1}{3}(2-\gamma)\left(\frac{\partial \phi_{1}}{\partial t}\right)^{2}\right] \\
& -R_{2}\left(\frac{\partial \phi_{1}}{\partial y}\right)^{3}-\chi_{2}\left(\frac{\partial \phi_{1}}{\partial y}\right)^{2} \frac{\partial^{2} \phi_{1}}{\partial y \partial t}, \quad \text { at } y=1
\end{aligned}
$$

Before carrying out the solution, we digress to discuss the limitations of the inviscid, irrotational gas assumption. Equations 3 and 14 show that $\vec{v}=0(\epsilon)$. Since $\tau=0(\vec{v})=0(\epsilon)$ and we are keeping terms of $0\left(\epsilon^{3}\right)$ in $\phi$ and $\overline{\overline{\vec{v}}}$, Eq. 2 shows that our analysis is valid when $\epsilon^{2}$ $>0\left(\mathrm{Re}^{-1}\right)$. For a duct with $d=1 \mathrm{ft}$. at room temperature $\left(c_{0}=1150 \mathrm{ft}\right.$. $/ \mathrm{sec} ., \nu=2.142 \times 10^{-4} \mathrm{ft} .{ }^{2} / \mathrm{sec}$. $), \operatorname{Re} \approx 5.4$ $\times 10^{6}$ and our analysis is valid for $\epsilon>4.3 \times 10^{-4}$ which corresponds to an SPL (sound pressure level) $\approx 130 \mathrm{~dB}$. For a duct with $d=1$ in., the analysis is valid for $\epsilon>1.5$ $\times 10^{-3}$ which corresponds to an SPL $\approx 140.5 \mathrm{~dB}$.

\section{SOLUTION}

We take the solution of Eq. 16 subject to the boundary conditions 17 and 18 in the form

$$
\phi_{1}=A\left(x_{2}\right) \cos \kappa y e^{i\left(k x_{0}-\omega t\right)}+\text { c. c. , }
$$

where c.c. stands for the complex conjugate of the preceding terms, $\omega=\omega^{*} d / c_{0}$ is the dimensionless frequency, and the real and imaginary parts $k_{0}$ and $\alpha_{0}$ of $k=k^{*} d$ are the dimensionless wavenumber and attenuation rate, respectively. Substituting Eq. 25 into Eqs. 16 and 18, we find that

$$
k^{2}=\omega^{2}-\kappa^{2},
$$

where $\kappa$ is a root of

$$
\kappa \tan \kappa=-i \omega\left(R_{0}-i \omega \chi_{0}\right)^{-1} \text {. }
$$

Note that the function $A\left(x_{2}\right)$ is undetermined to this order; it will be determined from the third-order problem.

Substituting for $\phi_{1}$ from Eq. 25 into Eqs. 19 and 21, we have

$$
\begin{aligned}
& \frac{\partial^{2} \phi_{2}}{\partial x_{0}^{2}}+\frac{\partial^{2} \phi_{2}}{\partial y^{2}}-\frac{\partial^{2} \phi_{2}}{\partial t^{2}}= 4 \kappa\left(\Gamma_{1} \kappa+\Gamma_{2} \cos 2 \kappa y\right) \\
& \times A^{2} \exp \left[2 i\left(k x_{0}-\omega t\right)\right]+\text { c. c. }, \\
& \frac{\partial \phi_{2}}{\partial t}+R_{0} \frac{\partial \phi_{2}}{\partial y}+\chi_{0} \frac{\partial^{2} \phi_{2}}{\partial y \partial t}=-\frac{1}{2} \kappa^{2} A^{2} \exp \left[2 i\left(k x_{0}-\omega t\right)\right] \\
&+\Gamma_{3} A \bar{A} \exp \left(-2 \alpha_{0} x_{0}\right)+\text { c. c. , at } y=1,
\end{aligned}
$$

where

$$
\begin{aligned}
& \Gamma_{1}=\frac{1}{4} i \omega\left[\frac{1}{2}(\gamma-1) \omega^{2}+k^{2}-\kappa^{2}\right] \kappa^{-2}, \\
& \Gamma_{2}=\frac{1}{8} i(\gamma+1) \omega^{2} \kappa^{-1}, \\
& \Gamma_{3}=-\frac{1}{2}\left(k \bar{k}-\omega^{2}\right) \cos \kappa \cos \bar{\kappa}-\frac{1}{2} \kappa \bar{\kappa} \sin \kappa \sin \bar{\kappa},
\end{aligned}
$$

where the overbar indicates the complex conjugate. The solution of the second-order problem (Eqs. 20, 28, and 29) depends on whether $\alpha_{0}$ is small or not. If $\alpha_{0}$ is not small,

$$
\begin{aligned}
\phi_{2}= & \left(\Gamma_{1}+\Gamma_{2} y \sin 2 \kappa y+\Gamma_{4} \cos 2 \kappa y\right) A^{2} \exp \left[2 i\left(k x_{0}-\omega t\right)\right] \\
& -\left(\Gamma_{3} / 2 \alpha_{0} R_{0} \sin 2 \alpha_{0}\right) A \bar{A} \cos 2 \alpha_{0} y \exp \left(-2 \alpha_{0} x_{0}\right)+\text { c. c. },
\end{aligned}
$$

where

$$
\begin{aligned}
\Gamma_{4}= & {\left[\frac{\mathrm{r}}{2} \kappa^{2}+\Gamma_{2}\left(R_{0}-2 i \chi_{0}\right)(\sin 2 \kappa+2 \kappa \cos 2 \kappa)\right.} \\
& \left.-2 i \omega\left(\Gamma_{1}+\Gamma_{2} \sin 2 \kappa\right)\right] \\
& \times\left\{2 i \omega \cos 2 \kappa+2 \kappa\left(R_{0}-2 i \omega \chi_{0}\right) \sin 2 \kappa\right\} .
\end{aligned}
$$

Note that Eq. 31 is not valid when $\alpha_{0}$ is small because the second term approaches $\infty$ as $\alpha_{0} \rightarrow 0$. When $\alpha_{0}^{2} \leq 0(\epsilon)$, $\exp \left(-\alpha_{0} x_{0}\right)$ must be considered slowly varying rather than fast varying when solving for $\phi_{2}$. The result is

$$
\begin{aligned}
\phi_{2}= & \left(\Gamma_{1}+\Gamma_{2} y \sin 2 \kappa y+\Gamma_{4} \cos 2 \kappa y\right) A^{2} \exp \left[2 i\left(k x_{0}-\omega t\right)\right] \\
& +\Gamma_{3} t A \bar{A} \exp \left(-2 \alpha_{0} x_{0}\right)+\text { c. c., when } \alpha_{0}^{2} \leq \epsilon .
\end{aligned}
$$

Substituting for $\phi_{1}$ and $\phi_{2}$ from Eqs. 25, and either Eq. 31 or Eq. 33 into Eqs. 22 and 24, we obtain

$$
\begin{aligned}
\frac{\partial^{2} \phi_{3}}{\partial x_{0}^{2}}+\frac{\partial^{2} \phi_{3}}{\partial y^{2}}-\frac{\partial^{2} \phi_{3}}{\partial t^{2}}= & {\left[-2 i k \frac{d A}{d x_{2}} \cos \kappa y\right.} \\
& \left.+F(y) A^{2} \bar{A} e^{-2 \alpha_{0} x_{0}}\right] e^{i\left(k x_{0}-\omega t\right)}+\text { c. c. }
\end{aligned}
$$$$
\text { +harmonics other than } \exp ( \pm i \omega t),(34)
$$$$
\frac{\partial \phi_{3}}{\partial t}+R_{0} \frac{\partial \phi_{3}}{\partial y}+\chi_{0} \frac{\partial^{2} \phi_{3}}{\partial y \partial t}=G A^{2} \bar{A} e^{-2 \alpha_{0} x_{0}} e^{i\left(k x_{0}-\omega t\right)}+\text { c. c. }
$$$$
\text { +harmonics other than } \exp ( \pm i \omega t) \text { at } y=1 \text {, }
$$

where $F$ and $G$ are defined in Appendix A. We seek a particular solution to Eqs. 23, 34, and 35 of the form

$$
\phi_{3}=\psi\left(y, x_{2}\right) \exp \left[i\left(k x_{0}-\omega t\right)\right] .
$$

Putting Eq. 36 into Eqs. 23, 34, and 35, we obtain 


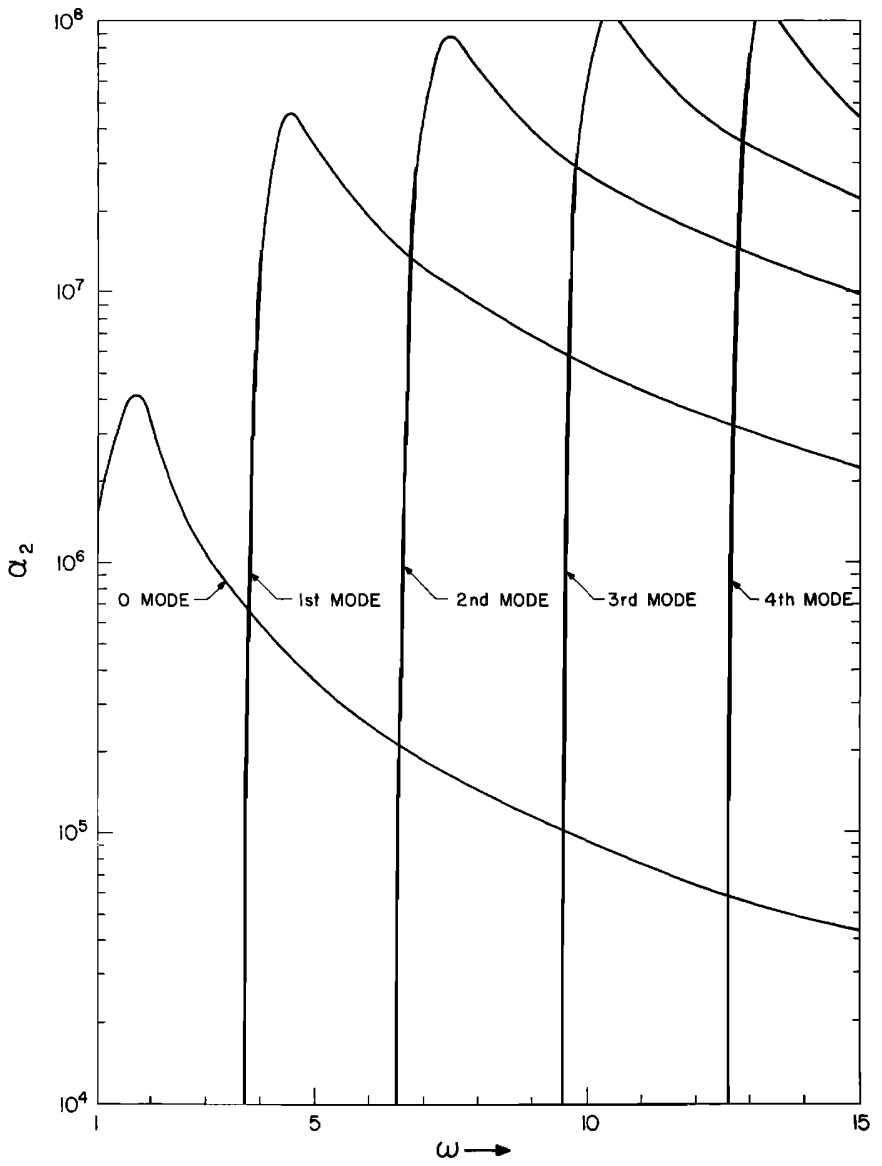

FIG. 2. Variation of $\alpha_{2}$ with $\omega$ for the lowest five modes $\left(R_{0}=0.1146, R_{2}=10^{6}, \hat{\chi}_{0}=0.12, \hat{\chi}_{2}=-10^{6}, f r=1000 \mathrm{~Hz}\right)$.

$$
\begin{array}{r}
\frac{\partial^{2} \psi}{\partial y^{2}}+\kappa^{2} \psi=-2 i k \frac{d A}{d x_{2}} \cos \kappa y+F(y) A^{2} \bar{A} \exp \left(-2 \alpha_{0} x_{0}\right), \\
\left(R_{0}-i \omega \chi_{0}\right) \frac{\partial \psi}{\partial y}-i \omega \psi=G A^{2} \bar{A} \exp \left(-2 \alpha_{0} x_{0}\right), \text { at } y=1, \\
\psi_{y}=0, \text { at } y=0 .
\end{array}
$$

The inhomogeneous Eqs. 37-39 have a solution if, and only if, their inhomogeneous parts are orthogonal to every solution of the adjoint homogeneous problem; that is,

$$
\begin{array}{r}
i k\left(1+\frac{\sin 2 \kappa}{2 \kappa}\right) \frac{d A}{d x_{2}}=\left(-\frac{G \cos \kappa}{R_{0}-i \omega \chi_{0}}+\int_{0}^{1} F(y) \cos \kappa y d y\right) \\
\times A^{2} \bar{A} e^{-2 \alpha x_{0}} .
\end{array}
$$

Solving for $d A / d x_{2}$, performing the integration, and replacing $x_{0}$ by $x$ and $x_{2}$ by $\epsilon^{2} x$, we obtain

$$
\frac{d A}{d x}=-\epsilon^{2}\left(\alpha_{2}+i k_{2}\right) A^{2} \bar{A} e^{-2 \alpha_{0} x},
$$

where $\alpha_{2}+i k_{2}$ is also defined in Appendix A. Letting $A$ $=a \exp \left(\alpha_{0} x+i \beta\right)$ with real $a$ and $\beta$ in Eq. 41 and separating real and imaginary parts, we get

$$
d a / d x=-\left(\alpha_{0}+\epsilon^{2} a^{2} \alpha_{2}\right) a,
$$

where $a$ is the amplitude of the wave.
Equation 42 shows that the attenuation rate of a given mode is the sum of two terms, an $\alpha_{0}$ which is independent of the amplitude, and an $\epsilon^{2} \alpha_{2} a^{2}$ which is a function of the amplitude. Thus, the nonlinearity increases or decreases the attenuation rate depending on whether $\alpha_{2}$ is positive or negative. Figure 2 shows the variation of $\alpha_{2}$ of the lowest five modes with frequency for $R_{0}=0.1146$, $\hat{\chi}_{0}=f_{r} d \chi_{0} / c_{0}=0.12, R_{2}=10^{6}, \hat{\chi}_{2}=\omega^{2} \chi_{2} c_{0} / f_{r} d=-10^{6}$, and a material reference frequency $f_{r}=1000 \mathrm{~Hz}$. For each mode, there is a threshold frequency above which the nonlinearity increases the attenuation $\left(\alpha_{2}>0\right)$ and below which the nonlinearity decreases the attenuation $\left(\alpha_{2}<0\right)$. Moreover, the threshold frequency increases with increasing mode number. This behavior is also demonstrated in Fig. 3, which shows the variation of the absorption coefficient (rate of change of $L$ with $x$ where $L$ is the sound pressure level) of the lowest four modes with frequency for a dimensionless particle velocity of 0.005 at the wall and the above material parameters except $R_{0}=1.0$ and $R_{2}=-\hat{\chi}_{2}=2 \times 10^{4}$. This figure shows also that for each mode there exists a threshold frequency below which the nonlinearity had an adverse effect and above which it has a favorable effect on the absorption coefficient, in qualitative agreement with the experimental findings of Zorumski and Parrott ${ }^{11}$ and Kurze and Allen. ${ }^{12}$ Moreover, the absorption coefficient of the

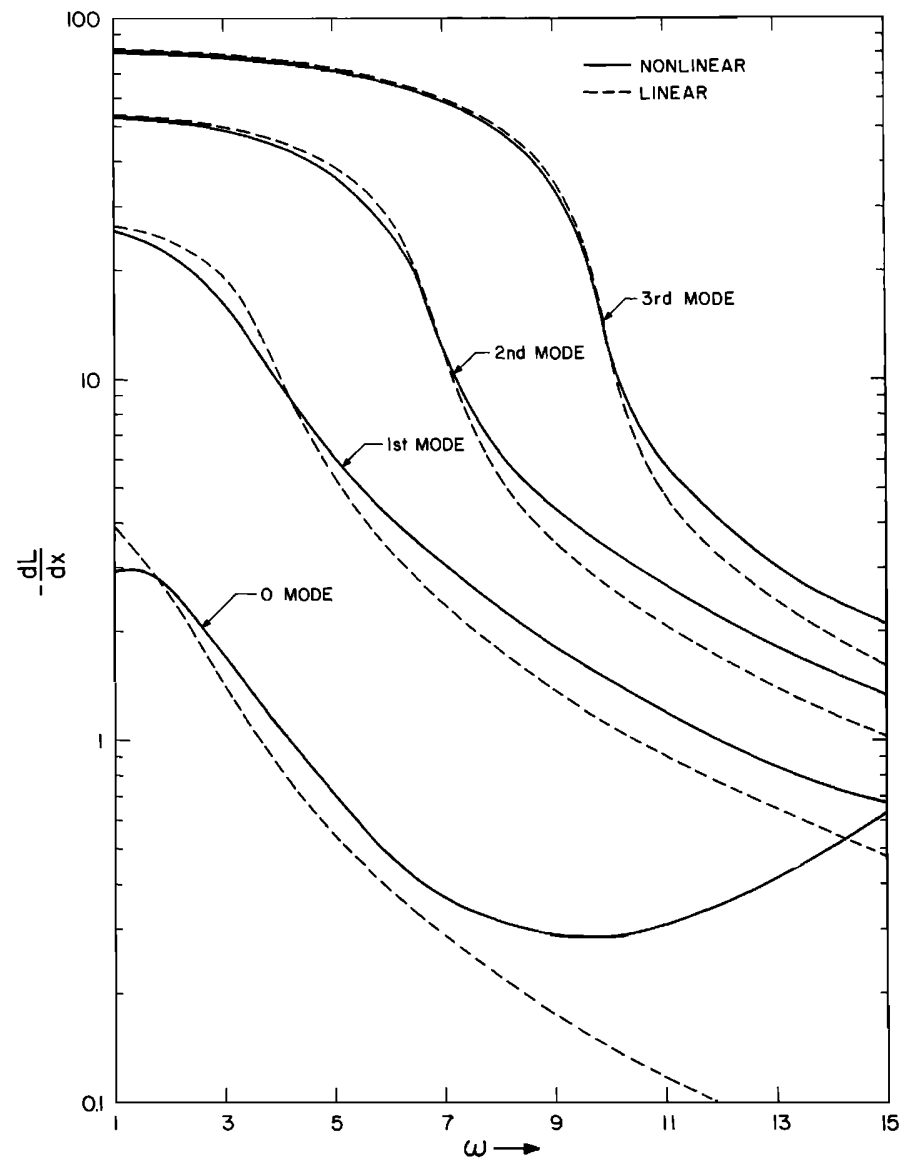

FIG. 3. Variation of the absorption coefficient of the lowest four modes with frequency for a wall particle, dimensionless velocity of 0.005 , and material parameters of Fig. 2, except $R_{0}=1.0$ and $R_{2}=-\hat{\chi}_{2}=2 \times 10^{4}$. 


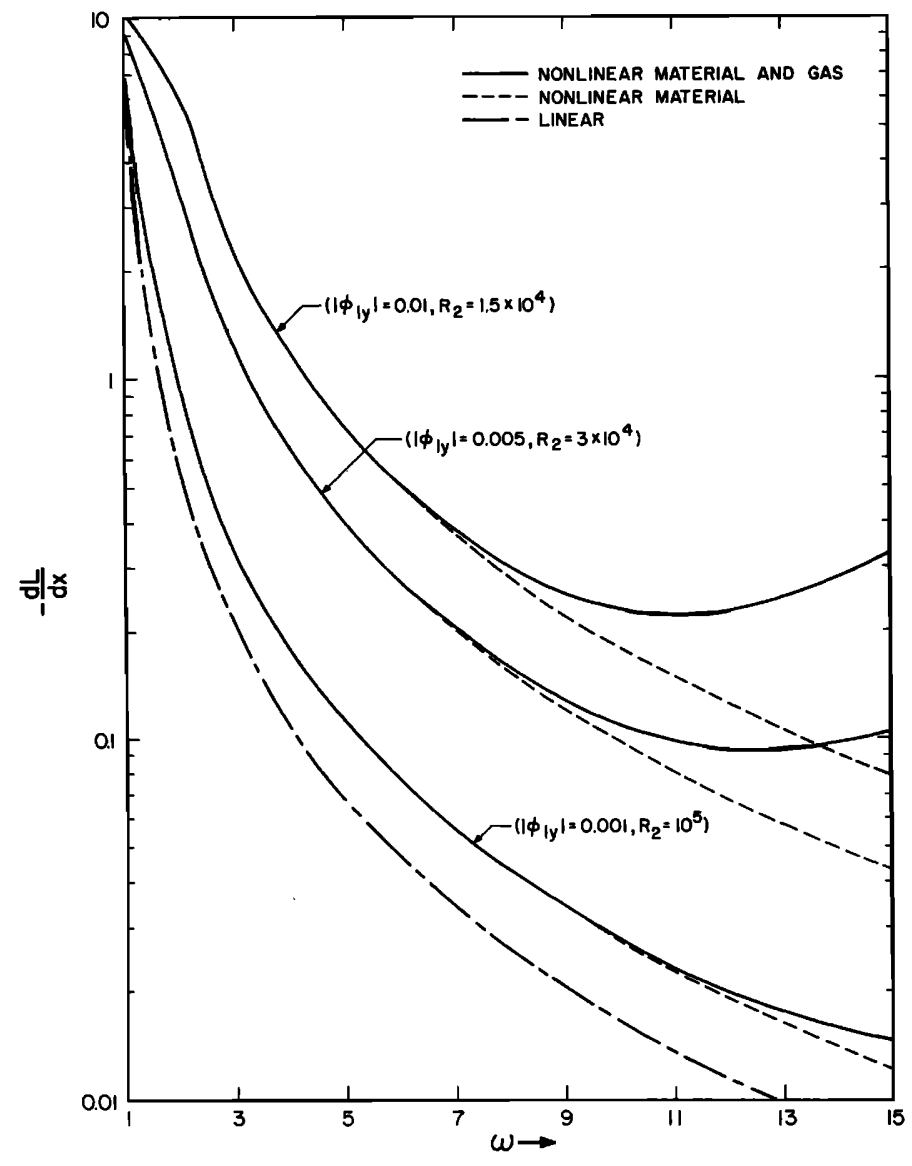

FIG. 4. Variation of the absorption coefficient of the lowest mode with frequency and nonlinearity $R_{0}=0.1146, \hat{x}_{0}=0.12$, $R_{2}=-\hat{\chi}_{2}$, and $f r=1000 \mathrm{~Hz}$.

lowest mode is much more affected by the nonlinearity than those of the higher modes, owing to its smaller linear absorption coefficient.

Figures 4 and 5 show the variation of the absorption coefficient of the lowest mode with frequency for the two linear resistances $R_{0}=0.1146$ and $R_{0}=1.0$. When the linear resistance is very much below. $\dot{R}_{m}$, the linear resistance corresponding to maximum absorption, such as $R_{0}=0.1146$, Fig. 4 shows that the nonlinearity increases the absorption coefficient because it pushes the total resistance toward $R_{m}$ (Fig. 6). On the other hand, when $R_{0}$ is below but near $R_{m}$ (such as $R_{0}=1.0$ ), Fig. 5 shows that the nonlinearity may have an adverse effect on the absorption, especially at low frequencies where the absorption coefficient may flatten. At high frequencies, the absorption coefficient broadens indicating a favorable effect.

Figures 4 and 5 show that the nonlinear effects of the material decrease with increasing sound frequency, whereas the nonlinear effects of the gas increase with increasing sound frequency. Moreover, the nearer $R_{0}$ to $R_{m}$ the larger is the effect of the gas nonlinearity.

\section{SUMMARY}

The method of multiple scales is used to determine quantitatively the nonlinear effects of the gas and the lin- ing material acoustic properties on the propagation and attenuation of sound waves in two-dimensional ducts. The results show that the nonlinear effects of the material decrease with frequency, in contrast with the nonlinear effects of the gas, which increase with frequency. The nonlinearity affects the lowest mode more than the higher modes. For each mode, there exists a threshold frequency below which the nonlinearity has an adverse effect and above which it has a favorable effect on the absorption coefficient, in qualitative agreement with the experimental observations of Zorumski and Parrott ${ }^{11}$ and Kurze and Allen. ${ }^{12}$ The threshold frequency increases with increasing mode number.

Note that the present analysis is valid only when the waves are dispersive; that is, $\kappa$ of Eqs. 26 and 27 is not very small.

\section{ACKNOWLEDGMENT}

This work was supported by NASA Langley Research Center under Grant NGR 47-004-109.

$$
\begin{aligned}
& \text { APPENDIX A } \\
& \begin{aligned}
F(y)= & \Lambda_{1} \cos \bar{\kappa} y+\Lambda_{2} \cos (2 \kappa-\bar{\kappa}) y+\Lambda_{3} \cos (2 \kappa+\bar{\kappa}) y \\
& +\Lambda_{4} y \sin (2 \kappa-\bar{\kappa}) y+\Lambda_{5} y \sin (2 \kappa+\bar{\kappa}) y+f(y),
\end{aligned}
\end{aligned}
$$

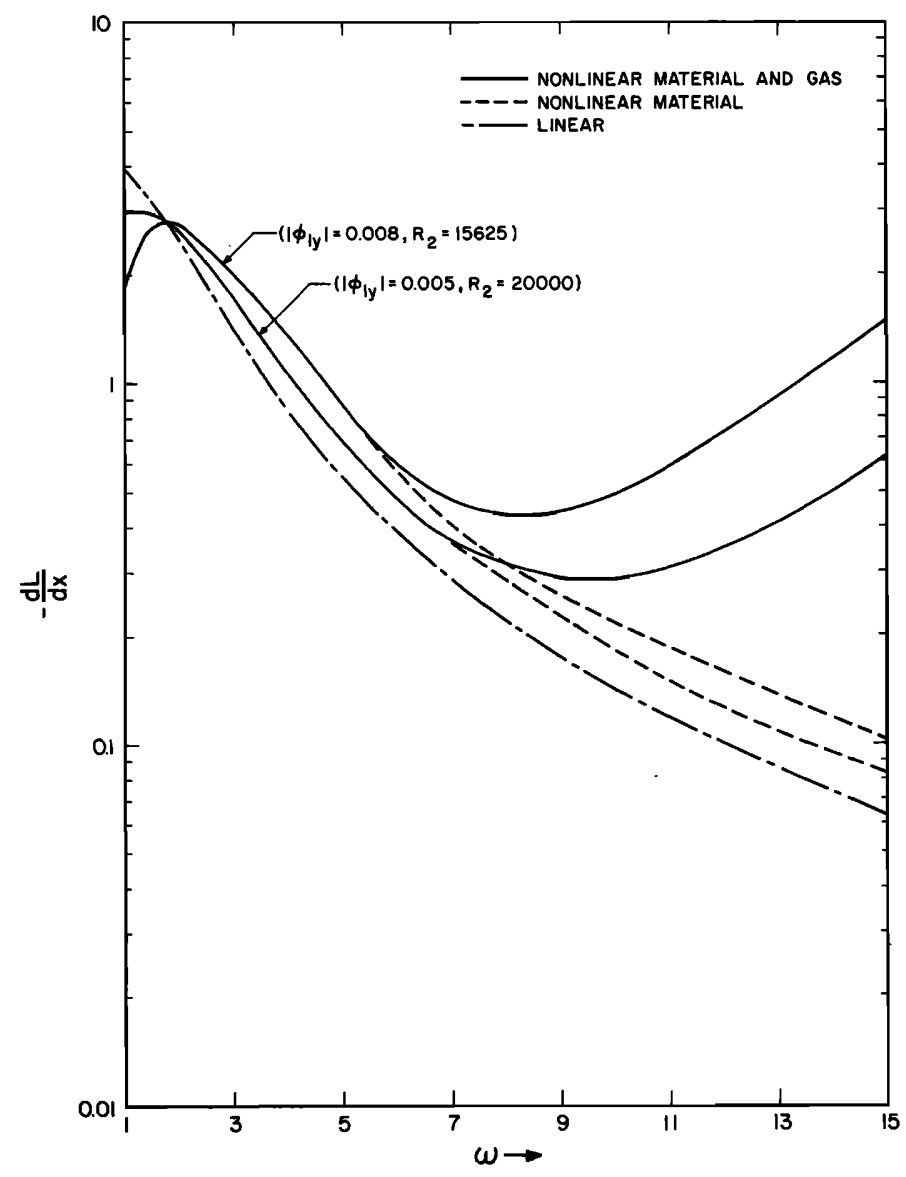

FIG. 5. Variation of the absorption coefficient of the lowest mode with frequency and nonlinearity for $R_{0}=1.0, \hat{\chi}_{0}=0.12$, $R_{2}=-\hat{\chi}_{2}$, and $f r=1000 \mathrm{~Hz}$. 


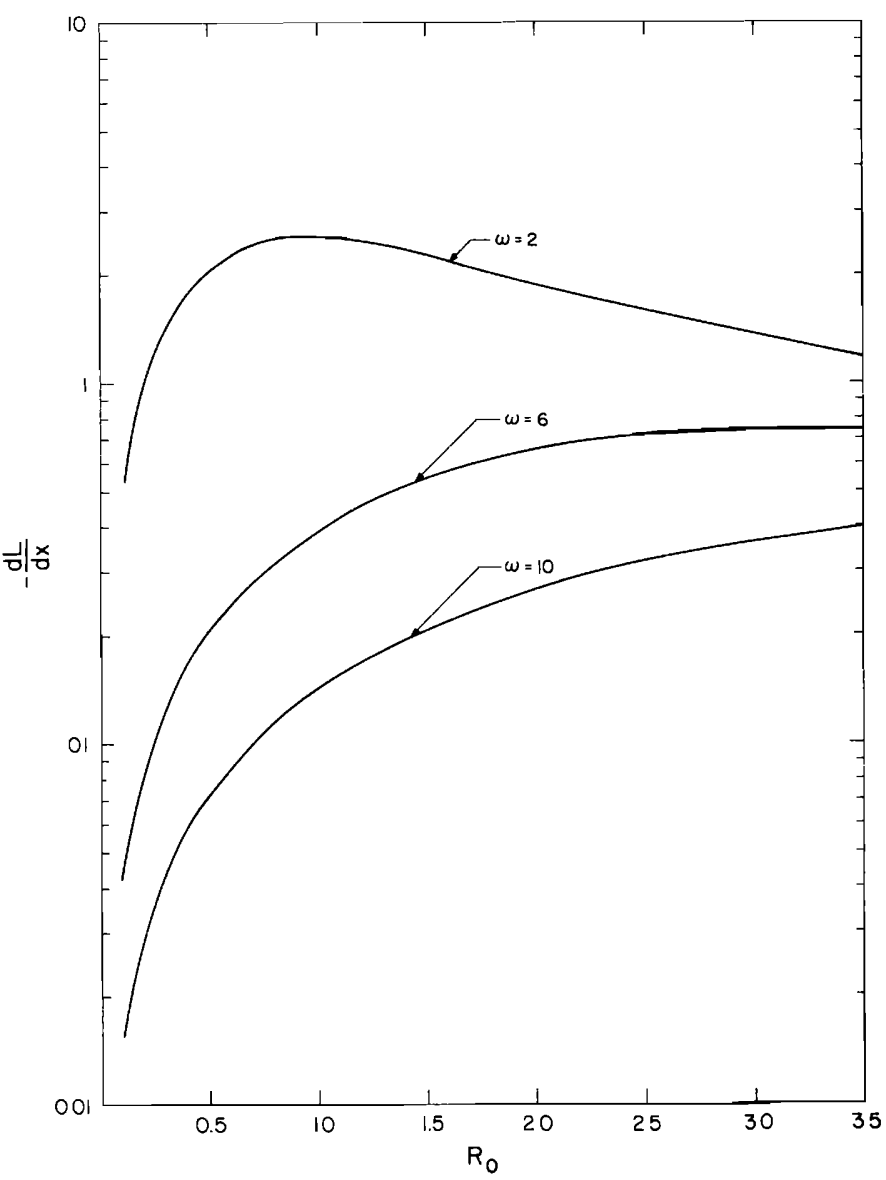

FIG. 6. Variation of the linear absorption coefficient of the lowest mode with the frequency and the linear resistance.

where

$$
\begin{aligned}
\Lambda_{1}= & \left.-2 i \omega \Gamma_{1}[\gamma-1) \omega^{2}+2 k \bar{k}\right]-\frac{1}{2}(\gamma-1)^{2} \omega^{4}+\frac{1}{2} k^{2} \bar{k}^{2}-\frac{1}{2} \kappa^{2} \bar{\kappa}^{2} \\
& -\frac{1}{2}(\gamma+1) k^{3} k-\frac{1}{2}(\gamma-3) k \bar{k} \kappa^{2}+\frac{3}{4}(\gamma-1) \omega^{2}\left(\kappa^{2}-k^{2}\right), \\
\Lambda_{2}= & -\frac{1}{8}(\gamma-1)(2 \gamma+1) \omega^{4}+i \omega \bar{\kappa} \Gamma_{2}-i \omega \Gamma_{4}\left[(\gamma-1) \omega^{2}\right. \\
& +2 k \bar{k}+2 \kappa \bar{\kappa}]+\frac{1}{4}(k \bar{k}+\kappa \bar{\kappa})\left[k \bar{k}+\kappa \bar{\kappa}-(\gamma+1) \omega^{2}\right], \\
\Lambda_{3}= & -\frac{1}{8}(\gamma-1)(2 \gamma+1) \omega^{4}-i \omega \bar{\kappa} \Gamma_{2}-i \omega \Gamma_{4}\left[(\gamma-1) \omega^{2}\right. \\
& +2 k \bar{k}-2 \kappa \bar{\kappa}]+\frac{1}{4}(k \bar{k}-\kappa \bar{\kappa})\left[k \bar{k}-\kappa \bar{\kappa}-(\gamma+1) \omega^{2}\right], \\
\Lambda_{4}= & -\Gamma_{2}\left[i(\gamma-1) \omega^{3}+2 i \omega k \bar{k}+2 i \omega \kappa \bar{\kappa}\right], \\
\Lambda_{5}= & -\Gamma_{2}\left[i(\gamma-1) \omega^{3}+2 i \omega k \bar{k}-2 i \omega \kappa \bar{\kappa}\right], \\
f(y)= & -2(\gamma-1) \omega^{2} \Gamma_{3} \cos \kappa y, \text { when } \alpha_{0}^{2} \leq 0(\epsilon), \\
= & \frac{2 i \omega \Gamma_{3}}{R_{0} \sin 2 \alpha_{0}}\left[(\kappa-i k) \cos \left(\kappa-2 \alpha_{0}\right) y\right. \\
& \left.-(\kappa+i k) \cos \left(\kappa+2 \alpha_{0}\right) y\right], \text { when } \alpha_{0}^{2}>0(\epsilon), \\
G=2 & \cos \bar{\kappa}\left(\Gamma_{1}+\Gamma_{2} \sin 2 \kappa+\Gamma_{4} \cos 2 \kappa\right)\left(\omega^{2}-k \bar{k}\right) \\
+ & \bar{\kappa} \sin \bar{\kappa}\left[(\sin 2 \kappa+2 \kappa \cos 2 \kappa) \Gamma_{2}-2 \kappa \Gamma_{4} \sin 2 \kappa\right] \\
- & i \omega \cos \kappa[k \bar{k} \cos \kappa \cos \bar{\kappa}+\kappa \bar{\kappa} \sin \kappa \sin \bar{\kappa}] \\
- & \frac{1}{2} i(\gamma-2) \omega^{3} \cos { }^{2} \kappa \cos \bar{\kappa}-2 i \omega \Gamma_{3} \cos \kappa \\
+ & \left(3 R_{2}-i \chi_{2} \omega\right) \kappa^{2} \bar{\kappa} \sin { }^{2} \kappa \sin \bar{\kappa} \\
&
\end{aligned}
$$

$$
\begin{aligned}
&+\frac{1}{2} i \omega \cos \bar{\kappa}\left(-\kappa^{2} \cos ^{2} \kappa+\kappa^{2} \sin ^{2} \kappa\right)+g, \\
& g=-2 i \omega \Gamma_{3} \cos \kappa, \text { when } \alpha_{0}^{2} \leq 0(\epsilon), \\
&=-\frac{2 \Gamma_{3}}{R_{0} \sin 2 \alpha_{0}}\left(i k \cos 2 \alpha_{0} \cos \kappa-\kappa \sin 2 \alpha_{0} \sin \kappa\right), \\
& \text { when } \alpha_{0}^{2}>0(\epsilon), \\
& \alpha_{2}+i k_{2}=\frac{-2 i \kappa Q_{3}}{k(2 \kappa+\sin 2 \kappa)},
\end{aligned}
$$

where

$$
\begin{aligned}
Q_{3}= & \frac{G \cos \kappa}{R_{0}-i \omega \chi_{0}}-\Lambda_{1}\left[\frac{\sin (\kappa+\bar{\kappa})}{2(\kappa+\bar{\kappa})}+\frac{\sin (\kappa-\bar{\kappa})}{2(\kappa-\bar{\kappa})}\right] \\
& -\Lambda_{2}\left[\frac{\sin (3 \kappa-\bar{\kappa})}{2(3 \kappa-\bar{\kappa})}+\frac{\sin (\kappa-\bar{\kappa})}{2(\kappa-\bar{\kappa})}\right] \\
& -\Lambda_{3}\left[\frac{\sin (3 \kappa+\bar{\kappa})}{2(3 \kappa+\bar{\kappa})}+\frac{\sin (\kappa+\bar{\kappa})}{2(\kappa+\bar{\kappa})}\right] \\
& -\Lambda_{4}\left[\frac{\sin (\kappa-\bar{\kappa})}{2(\kappa-\bar{\kappa})^{2}}-\frac{\cos (\kappa-\bar{\kappa})}{2(\kappa-\bar{\kappa})}-\frac{\cos (3 \kappa-\bar{\kappa})}{2(3 \kappa-\bar{\kappa})}+\frac{\sin (3 \kappa-\bar{\kappa})}{2(3 \kappa-\bar{\kappa}) 2}\right] \\
& -\Lambda_{5}\left[\frac{\sin (\kappa+\bar{\kappa})}{2(\kappa+\bar{\kappa})^{2}}+\frac{\sin (3 \kappa+\bar{\kappa})}{2(3 \kappa+\bar{\kappa}) 2}-\frac{\cos (3 \kappa+\bar{\kappa})}{2(3 \kappa+\bar{\kappa})}-\frac{\cos (\kappa+\bar{\kappa})}{2(\kappa+\bar{\kappa})}\right]+q, \quad(\mathrm{~A} 11) \\
q= & (\gamma-1) \omega^{2} \Gamma_{3}\left(1+\frac{\sin 2 \kappa}{2 \kappa}\right), \text { when } \alpha_{0}^{2} \leq 0(\epsilon), \\
& \left.-\frac{2 i k \sin 2 \alpha_{0}}{\alpha_{0}}\right], \text { when } \alpha_{0}^{2}>0(\epsilon) \cdot \\
= & -\frac{i \omega \Gamma_{3}}{2 R_{0} \sin 2 \alpha_{0}}\left[\frac{(\kappa-i k) \sin 2\left(\kappa-\alpha_{0}\right)}{\kappa-\alpha_{0}}-\frac{(\kappa+i k) \sin 2\left(\kappa+\alpha_{0}\right)}{\kappa+\alpha_{0}}\right. \\
& \\
&
\end{aligned}
$$

${ }^{1}$ R. D. Fay, "Plane Sound Waves of Finite Amplitude," J. Acoust. Soc. Am. 3, 222-241 (1931).

${ }^{2}$ G. E. Fubini, "Anomalie nella propagazione di onde acustische die grande ampiezza," Alta Freq. 4, 530-581 (1935).

${ }^{3}$ D. T. Blackstock, "Connection between the Fay and Fubini Solutions for Plane Sound Waves of Finite Amplitude," J. Acoust. Soc. Am. 39, 1019-1026 (1965).

${ }^{4}$ A. B. Coppens, "Theoretical Study of Finite Amplitude Traveling Waves in Rigid-Walled Ducts: Behavior for Strengths Precluding Shock Formation," J. Acoust. Soc. Am. 49, 306318 (1971).

${ }^{5}$ F. M. Pestorius and D. T. Blackstock, "Nonlinear Distortion in the Propagation of Intense Acoustic Noise," Interagency Symposium on University Research in Transportation Noise, 565-577 (1973).

${ }^{6}$ S. H. Maslen and F. K. Moore, "On Strong Transverse Waves Without Shocks in a Circular Cylinder," J. Aerospace Sci. 23, 583-593 (1956).

${ }^{7}$ A. H. Nayfeh, Perturbation Methods (Wiley-Interscience, New York, 1973).

${ }^{8}$ S. H. Burns, "Finite Amplitude Distortion in Air at High Acoustic Pressures," J. Acoust. Soc. Am. 41, 1157-1169 (1967).

${ }^{9}$ J. B. Keller and M. H. Millman, "Finite Amplitude Sound Wave Propagation in a Waveguide," J. Acoust. Soc. Am. 49, 329-333 (1971). 
${ }^{10} \mathrm{~J}$. L. Peube and J. Chesseriaux, "Nonlinear Acoustics in Ducts with Varying Cross Section," J. Sound Vib. 27, 533548 (1973).

${ }^{11}$ W. E. Zorumski and T. L. Parrott, "Nonlinear Acoustic Theory for Thin Porous Sheets," NASA SP-189, 17-27 (1968).

${ }^{12}$ U. J. Kurze and C. H. Allen, "Influence of Flow and High Sound Level on the Attenuation in a Lined Duct," J. Acoust. Soc. Am. 49, 1643-1653 (1971).

${ }^{13} \mathrm{U}$. Ingard, "Nonlinear Attenuation of Sound in a Duct," J. Acoust. Soc. Am. 43, 167-168 (1968).
${ }^{14} \mathrm{U}$. Ingard, "Absorption Characteristics of Nonlinear Acoustic Resonators," J. Acoust. Soc. Am. 44, 1155-1156 (1968).

${ }^{15}$ M. A. Isakovich, "Nonlinear Effects Involved in Certain Acoustical Problems," Sov. Phys. -Acoust. 6, 321-325 (1961).

${ }^{16}$ T. H. Melling, "The Acoustic Impedance of Perforates at Medium and High Sound Pressure Levels," J. Sound Vib. 29, 1-65 (1973).

${ }^{17}$ W. E. Zorumski and T. L. Parrott, "Non-linear Acoustic Theory for Rigid Porous Materials," NASA TN-6196 (1971). 